\title{
Compact Genetic Algorithm for Active Interval Scheduling in Hierarchical Sensor Networks
}

\author{
Ming-Hui Jin ${ }^{1}$, Cheng-Yan Kao ${ }^{4}$, \\ Yu-Cheng Huang ${ }^{5}$ \\ Dep. of Computer Science and \\ Information Engineering, National \\ Taiwan University, Taipei, Taiwan \\ 1mhjin@mems.iam.ntu.edu.tw \\ 4cykao@csie.ntu.edu.tw \\ 5r91021@csie.ntu.edu.tw
}

\author{
D. Frank Hsu
}

Dep. of Computer and

Information Sciences,

Fordham University, LL813

New York, NY 10023

hsu@trill.cis.fordham.edu
Ren-Guey Lee

Dep. of Electronic

Engineering, National

Taipei University of

Technology, Taipei,

Taiwan

evans@ntut.edu.tw

\author{
Chih-Kung Lee \\ Inst. of Applied \\ Mechanics, National \\ Taiwan University, Taipei, \\ Taiwan
}

cklee@mems.iam.ntu.edu.tw

\begin{abstract}
This paper introduces a novel scheduling problem called the active interval scheduling problem in hierarchical wireless sensor networks for long-term periodical monitoring applications. To improve the report sensitivity of the hierarchical wireless sensor networks, an efficient scheduling algorithm is desired. In this paper, we propose a compact genetic algorithm (CGA) to optimize the solution quality for sensor network maintenance. The experimental result shows that the proposed CGA brings better solutions in acceptable calculation time.
\end{abstract}

\section{Categories \& Subject Descriptors: Computer Application $\rightarrow$} MISCELLANEOUS

\section{General Terms: Algorithm}

Keywords: Sensor Network, Active Interval Scheduling Problem

\section{NETWORK ARCHITECTURE}

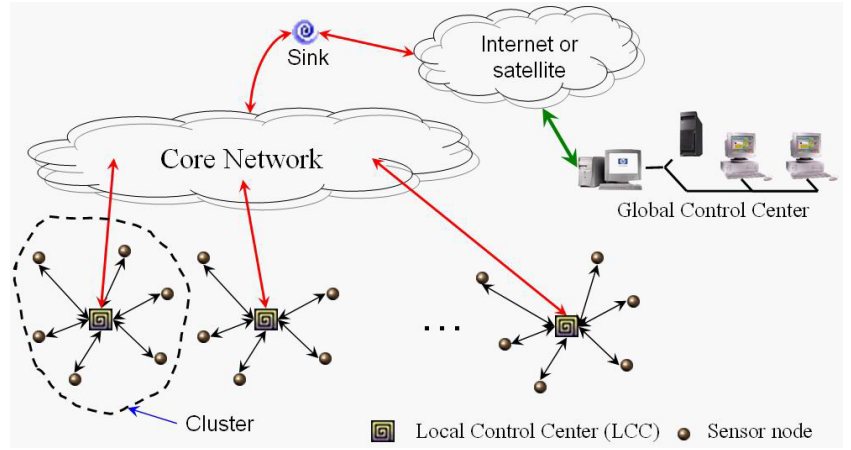

Figure 1. The network architecture for sensor networks with immobile sensors.

Figure 1 shows the network architecture proposed in [1]. In this architecture, the sensor network is partitioned into several clusters. Each cluster contains several sensor nodes and a local control center (LCC). A sensor node has capability to detect and then reports the detection results to its LCC. The detection results are then routed back to the sink through the Core Network constructed by only the LCCs. The sink may communicate with the global control center (GCC) via Internet or satellite. The sensor nodes are immobile in this study. In each cluster, the LCC applies the polling protocol to communicate with all its sensor nodes.

Copyright is held by the author/owner(s).

GECCO'05, June 25-29, 2005, Washington, DC, USA.

ACM 1-59593-010-8/05/0006.
In [1], each cluster is assumed to be active periodically and the entire clusters apply the same period. The period for all the clusters is said to be the detection cycle and the length of detection cycle is denoted as $l d c$. Each cluster is allowed to be active in the same period in all the detection cycle, and the period is called the active interval of the cluster.

\section{THE ACTIVE INTERVAL SCHEDULING PROBLEM}

We adopt the following definitions proposed in [1] as following.

$D$ 1: $C L=\left\{C_{1}, C_{2}, \ldots, C_{n}\right\}$ be the set of all clusters, where $n$ is the number of cluster nodes in the core network.

$D$ 2: For each $1 \leq i \leq n$, cluster $C_{i}$ contains $s_{i}$ sensors.

$D$ 3: The active interval of $C_{i}$ is denoted as $\left(t_{s}(i), t_{e}(i)\right)$.

$D$ 4: Two clusters $C_{i}$ and $C_{j}$ are adjacent if any sensor node in $C_{i}$ can receive any broadcasted messages from the $\mathrm{LCC}$ of $C_{j}$ to the sensor nodes of $C_{j}$.

$D$ 5: For two different clusters $C_{i}$ and $C_{j}, R_{i j}=1$ if $C_{i}$ and $C_{j}$ are adjacent and $R_{i j}=0$ otherwise.

$D$ 6: The minimal feasible value of $l d c$ is denoted as $l d c_{\text {min }}$.

Since $l d c_{\min }=\min \left\{t_{e}(i) \mid C_{i} \in C L\right\}$, and the network requires adjacent clusters should not be active simultaneously. Therefore, in [1], the cost model for $l d c_{\text {min }}$ minimization is stated as follow.

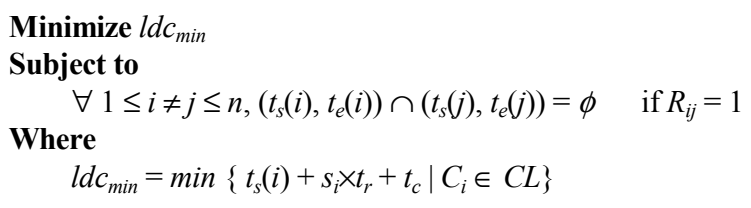

\section{THE PROPOSED COMPACT GENETIC ALGORITHM}

The methodology of the algorithm design is to classified all the clusters into $m$ sets $V_{1}, \ldots, V_{m}$. The set $V_{i}$ is called the $i^{\text {th }}$ selection of the solution. The clusters in the same selection can be active simultaneously. The active interval of each cluster in the $i^{\text {th }}$ selection is a sub-interval of $\left(t_{i-1}, t_{i}\right)$, and hence $l d c_{\min }=t_{m}$.

Assume that $i-1$ selections have been determined and there are $n(i)$ clusters $C L(i)=\left\{C_{\pi(\mathrm{i}, 1)}, \ldots, C_{\pi(i, n(i))}\right\} \subseteq C L$ which are not classified to any selection. In this situation, we make the following definitions

D. 6. A chromosome is defined to be a vector of ordered pairs $<\left(u_{1}\right.$, $\left.p_{1}\right), \ldots,\left(u_{n(i)}, p_{n(i)}\right)>$. For each $1 \leq j \leq n(i), u_{j}=1$ implies that the cluster $C_{\pi(i, j)}$ should be selected in the $i^{\text {th }}$ selection and $u_{j}=0$ otherwise. Besides, $p_{j}$ is denoted as the possibility of selecting 
the cluster $C_{\pi(i, j)}$. And we say that the chromosome selects the cluster $C_{\pi(i, j)}$ iff $u_{j}=1$.

D. 7. For chromosome $X=<\left(u_{1}, p_{1}\right), \ldots,\left(u_{n(i)}, p_{n(i)}\right)>$, the vector $\left.<u_{1}, \ldots, u_{n(i)}\right\rangle$ is called the selection vector of $X$, the vector $\left\langle p_{1}, \ldots, p_{n(i)}\right\rangle$ is called the probability vector of $X$ and the set $\{1$ $\left.\leq j \leq n(i) \mid u_{j}=1\right\}$ is called the selected index of $X$.

D. 8. A chromosome is said to be feasible if, for each two different clusters $C_{\pi(i, x)}$ and $C_{\pi(i, y)}$ in $C L(i), C_{\pi(i, x)}$ and $C_{\pi(i, y)}$ are adjacent implies $\left(u_{x}, u_{y}\right) \neq(1,1)$.

D. 9. A chromosome $\mathrm{X}$ is said to be a corrected chromosome of chromosome $\mathrm{Y}$ if $\mathrm{X}$ is a feasible and the selected index of $\mathrm{X}$ is a subset of the selected index of Y. Besides, we denote CC(Y) to be the set of all corrected chromosomes of Y. A correctness chromosome $x$ of chromosome $X$ is said to be a maximal correctness chromosome of $\mathrm{X}$ if, $\forall x \in \mathrm{CC}(\mathrm{X})$, the number of elements of the selected index of $y$ is greater than or equal to the number of elements of the selected index of $x$.

All the chromosomes in the each population are generated by two steps. First, determines the probability vector. Second, applies the probability vector to randomly generate the selection vector. That is, the probability of $u_{j}=1$ is $p_{j}$. All the chromosomes in the initial generation own the same probability vector $\left\langle p_{1}, \ldots, p_{n(i)}>\right.$ with $p=p_{j}$ for all $1 \leq j \leq n(i)$. The parameter $p$ is called the initial probability vector generator (IPVG).

\subsection{The Genetic Operators}

The competition operator generates two chromosomes called winner and loser, where the winner is the one with higher fitness. The competition operator applies the following steps to determine the winner and loser.

Step 1. Randomly generate a maximal correctness chromosome of $X$.

Step 2. Given the condition that the previous $i-1$ selections have been determined and all the clusters which are selected by the maximal correctness chromosome of $X$ have be classified into the $i^{\text {th }}$ selection, apply the Algorithm 1 in [1] to generate an active interval schedule $A I S_{X}$.

Step 3. Apply Step 1 and Step 2 to generate an $A I S_{Y}$ for $Y$

If the cost of $A I S_{X}$ is lower than the cost of $A I S_{Y}$, then set winner to be $X$ and then set loser to be $Y$.

The crossover operator applies the procedures below to generate a new chromosome from two given chromosomes $X$ and $Y$.

Step 1. Apply the competition operator to derive the two chromosomes winner and loser.

Step 2. Let $j=1$

Step 3. If $j>n(i)$, terminates this procedure

Step 4. Let $P_{\text {win }}(j)=$ the probability that the winner selects the cluster $C_{\pi(i, j)}, P_{\text {lose }}(j)=$ the probability that the loser selects the cluster $C_{\pi(i, j)}, P_{\text {min }}(j)=\min \left\{P_{\text {win }}(j), P_{\text {lose }}(j)\right\}$ and $P_{\max }(j)=$ $\max \left\{P_{\text {win }}(j), P_{\text {lose }}(j)\right\}$.

Step 5. If the winner selects the cluster $C_{\pi(i, j)}$, go to step 8 .

Step 6. If the loser selects the cluster $C_{\pi(i, j)}$, then set the probability that the new chromosome will select $C_{\pi(i, j)}$ to be $P_{\min }(j)-1 / S$, where $S$ is the population size. Otherwise, set the probability that the new chromosome will select to be $P_{\text {win }}(j)$.

Step 7. $j=j+1$ and then go to step 3 .

Step 8. If the loser selects the cluster $C_{\pi(i, j)}$, then set the probability that the new chromosome will select $C_{\pi(i, j)}$ to be $P_{\text {win }}(j)$.
Otherwise, set the probability that the new chromosome will select to be $P_{\max }(j)+1 / S$.

Step 9. $j=j+1$ and then go to step 3 .

\subsection{The Proposed Algorithm}

The proposed compact genetic algorithm for generating the $i^{\text {th }}$ selection is stated as follow, where $G$ in Step 3 is the maximal generation of this algorithm.

Step 1. Let $g=1$.

Step 2. Randomly generate $S$ chromosomes each one of which has the same probability $p$ to select all the remaining clusters.

Step 3. If $g>G$, go to step 8 .

Step 4. Let $h=1$

Step 5. Randomly selects two chromosomes $X$ and $Y$ generated in the $g^{\text {th }}$ generation, and then applies the crossover operator to generate a new chromosome in the $(g+1)^{\text {th }}$ generation

Step 6. Let $h=h+1$. If $h \leq S$, go to step 5 .

Step 7. If there are any chromosomes in the $(g+1)^{\text {th }}$ generation which definitely select or definitely not select a certain cluster, then let $g=g+1$ and then go to step 8. Otherwise, let $g=g+1$ and then go to step 3 .

Step 8. Apply the Algorithm 1 of [1] to generate an active interval schedule for each chromosome in the $g^{\text {th }}$ generation. Select the chromosome whose derived active interval schedule has lowest cost and then terminates this algorithm.

\section{EXPERIMENTS AND COMPARISONS}

The experiments were performed on an Pentium IV $2.6 \mathrm{GHz}$ PC with 1 GB memory running windows XP operation system. In all the experiments, the population size $S$ of solving each problem is 100 , the maximal generation of each experiment $G$ is 60000 and the IPVG is 0.5 . Five benchmark problems proposed in [1] are examined in the experiments.

\subsection{Experimental Results and Comparisons}

\begin{tabular}{|c|c|c|c|c|}
\hline \multirow{2}{*}{ Problem } & \multirow{2}{*}{$\begin{array}{c}\text { Algorithm } \\
1 \text { in [1] }\end{array}$} & \multirow{2}{*}{$\begin{array}{c}\text { Algorithm } \\
2 \text { in [1] }\end{array}$} & \multicolumn{2}{|c|}{ CGA } \\
\hline & & & Cost & CPU time \\
\hline 1 & 344 & 328 & 324 & 0.37 \\
\hline 2 & 566 & 548 & 532 & 1.54 \\
\hline 3 & 564 & 557 & 544 & 2.79 \\
\hline 4 & 791 & 773 & 752 & 5.59 \\
\hline 5 & 1396 & 1361 & 1138 & 10.29 \\
\hline
\end{tabular}

Table 1 compares the cost of the best solution of problem 1 to problem 5 derived by the greedy algorithms (Algorithm 1 and Algorithm 2 in [1]) and the compact genetic algorithm. The CPU times for generating the best solutions by the greedy algorithms are not presented in Table 1 since they are too small and hence been ignored in this table. The unit of the CPU time is 1 second. The experimental result in Table 1 shows that the CGA can further improve the solution quality in acceptable calculation time.

\section{REFERENCES}

[1] Ming-Hui Jin, Yu-Cheng Huang, D. Frank Hsu, Cheng-Yan Kao, You-Rui Wu, and Chih-Kung Lee, "On Active Interval Scheduling in Static Sensor Networks", Proceeding of IASTED International Conference on Communication System and Applications, July 2004. pp. $126-131$. 